\title{
THE BUSINESS OF CREATING GAMES
}

\author{
How to Make Positive and Effective in Multiple Area of a Business by Creating Games
}

\author{
Arsa Widitiarsa Utoyo, S.T., M.Sn. \\ Bina Nusantara University \\ School of Design \\ Jakarta, Indonesia \\ arsa_w@binus.ac.id
}

\begin{abstract}
The use of digital games and gamification has showed potential to improve many aspects of how businesses provide education and communicate with consumers. Nevertheless, there is still a requirement for better considerate of how the adoption of games and gamification would influence the process of decisionmaking for customer and organizations across different industry. This article provides a structured review of existing literature on the use of games in the business ecosystem, and seeks to consolidate findings to address research questions regarding their perception, proven efficiency, and identifies key areas for future business. The findings highlight that serious games can have positive and effective impacts in multiple areas of business, including education, decision-support, marketing and consumer outreach. They also emphasize the challenges and pitfalls of affecting serious games and gamification principles within a business context, and discuss the implications of development and evaluation methodologies on the success of a game-based solution.
\end{abstract}

Keywords: Serious Games, Game Based Learning, Business Game, Games

\section{INTRODUCTION}

Today, modern businesses in any industry are frequently faced with challenges such as rapidly evolving marketplaces, shifting labor markets, and the need to reach consumers who are increasingly engaging through a wide range of digital media. Focusing these challenges requires a wide range of skills from both senior and front-line staff, in-turn needing innovative and effective guidance tools such as serious games, gamification applications to aid staff at all levels of an organization as they adapt in response to emerging challenges. Hence it is important to analyze the benefits and pitfalls of these technologies in to demonstrate the impact that such technologies can have in an organization.

Games are the future. From education and business, to art and entertainment, game industry brings together the most innovative and creative minds to create the most engaging, immersive and breathtaking experiences we've ever seen. Games are a fundamental part of human existence. The phrasing of games has insinuated itself into our language to refer to activities that are not truly games. We play along with activities we find distasteful. We play ball with those who require our cooperation. We play games when we are disingenuous. A willing participant is game for the enterprise. This broad penetration of gaming concepts into the entire spectrum of human experience presents us with two potential barriers to understanding games.

The brilliant developers, designers and creators behind our games have and will continue to push the packet, driving extraordinary leaps in technology impacting everyday life for years to come. Most game begin with a single idea, the idea can revolve around character, gameplay, philosophy, or new technology. The idea might come from a friend, coworker, or publisher of from deep in your own subconscious. Sometimes the idea is completely original, but more often it builds on the work of game designers who have gone before. The goal of the first phase of development is to come up with your idea, which will eventually evolve into the high concept of the game. Here are some sample high concepts:

1. A busty female archaeologist pursues ancient treasure (Tomb Raider)

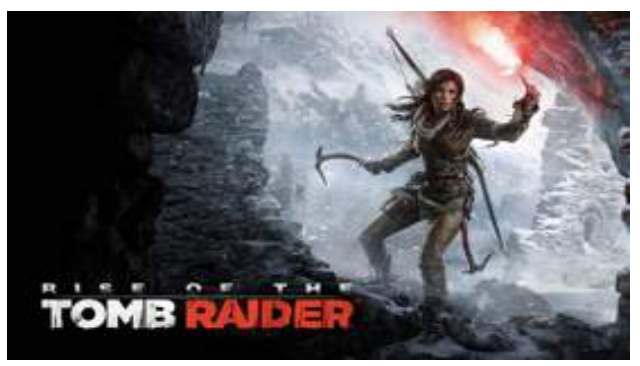

Figure 1 Rise of The Tomb Raider (https://www.tombraider.com/en-us)

2. Ping pong on the computer (Pong)

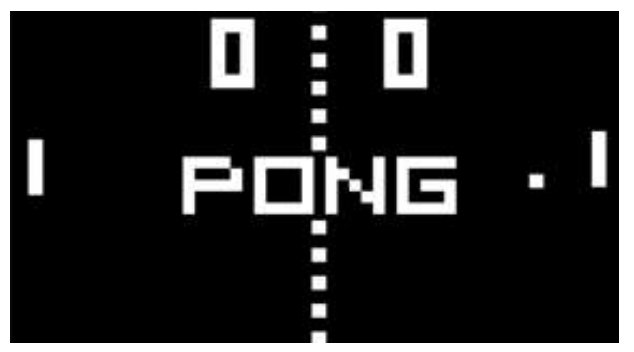

Figure 2 Pong (http://www.ponggame.org/) 
3. An ordinary technician battles trans dimensional monster after an accident at a secret facility (Half life)

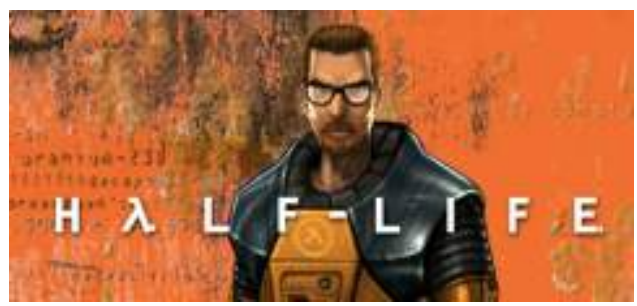

Figure 3 Half Life (http://orange.half-life2.com/)

4. A street racing game where you drive a getaway car for the mob (Driver)

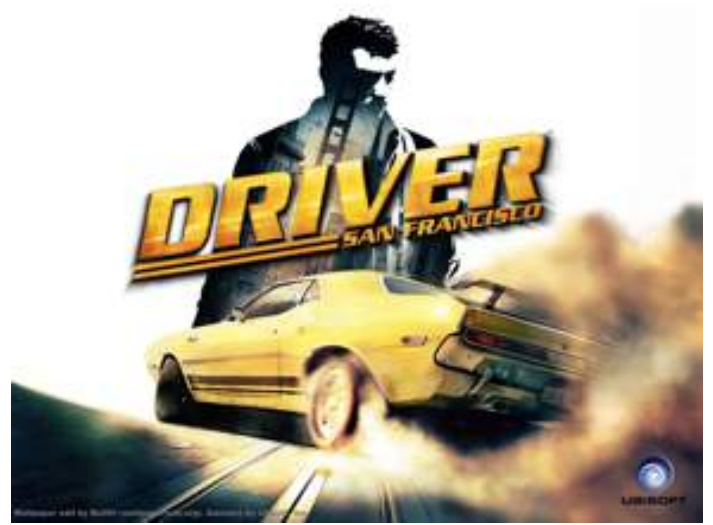

Figure 3 Driver San Francisco (https://www.ubisoft.com/enUS/game/driver-san-francisco/)

The high concept might fall neatly into an existing genre, but more and more gamers are hybrids, games that combine elements from different categories. These are the game genre that probably can enjoy playing:

Adventure games a story based games that usually rely on puzzle solving to move the action along. Adventure are generally not real time games, unless they are an action adventure hybrid. The original adventures are parser based; that is, they accept simple sentence commands from the keyboard.

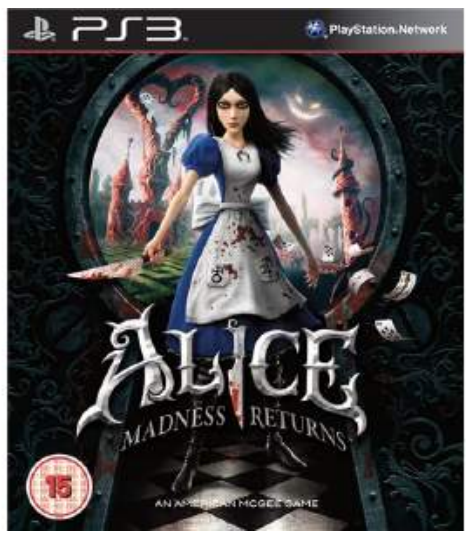

Figure 4 Alice Madness Returns (http://www2.ea.com/alice)
Vol. 01, No. 02, 2016

Strategic game is a game (e.g. video or board game) in which the players' uncovered, and often autonomous decisionmaking skills have a high significance in determining the outcome. Almost all strategy games require internal decision tree style thinking, and typically very high situational awareness.

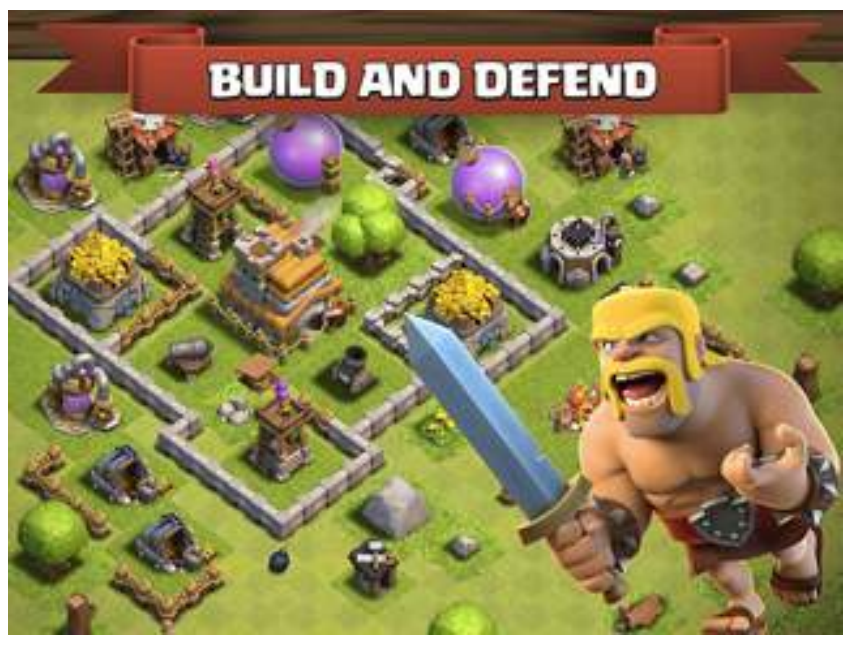

Figure 7 Clash of Clan (https://clashofclans.com/)

A simulation video game describes a diverse supercategory of video games, generally designed to closely simulate aspects of a real or fictional reality. A simulation game attempts to copy various activities from real life in the form of a game for various purposes such as training, analysis, or prediction.

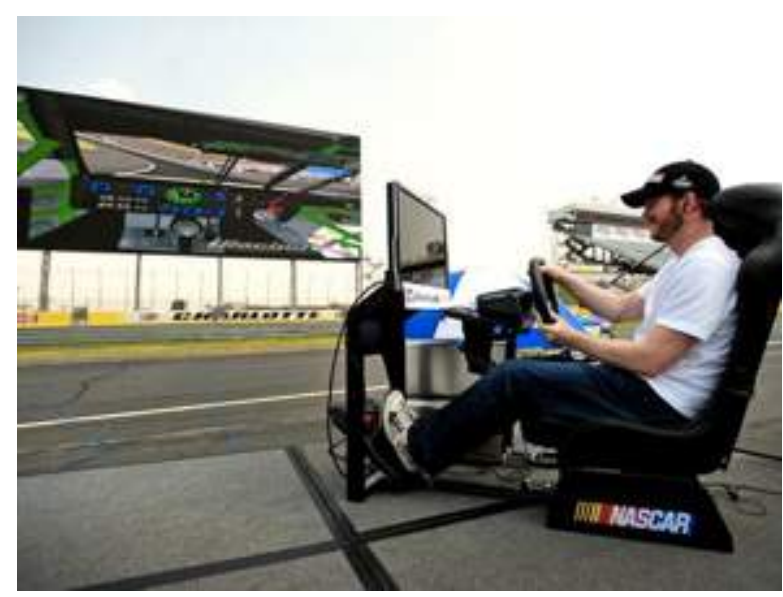

Figure 8 Nascar Simulation (http://www.iracing.com/)

A sports game is a video game that simulates the practice of sports. Most sports have been recreated with a game, including team sports, track and field, extreme sports and combat sports. 


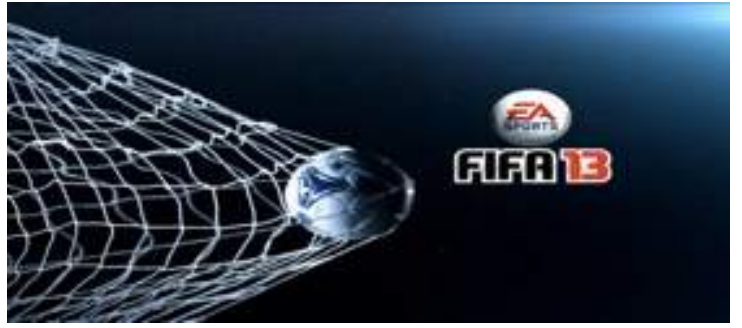

Figure 8 Fifa Soccer 2013 (https://www.ea.com/fifa)

A fighting game is a genre of video game in which a gamer battles against another character controlled by another gamer or the game's artificial intelligence (AI). Fighting games often feature special moves that are triggered through rapid sequences of button presses or associated mouse or joystick movements.

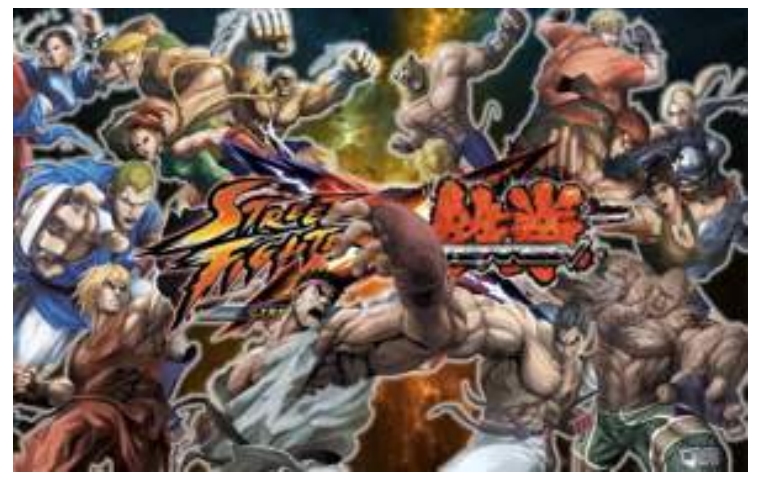

Figure 9 Street Fighter vs Tekken (http://www.gamespot.com/streetfighter-x-tekken/)

A casual game is a video game targeted at or used by a mass audience of casual gamers. Casual games can have any type of gameplay, and fit in any genre. They are typically distinguished by their simple rules and lack of commitment required in contrast to more complex hardcore games.

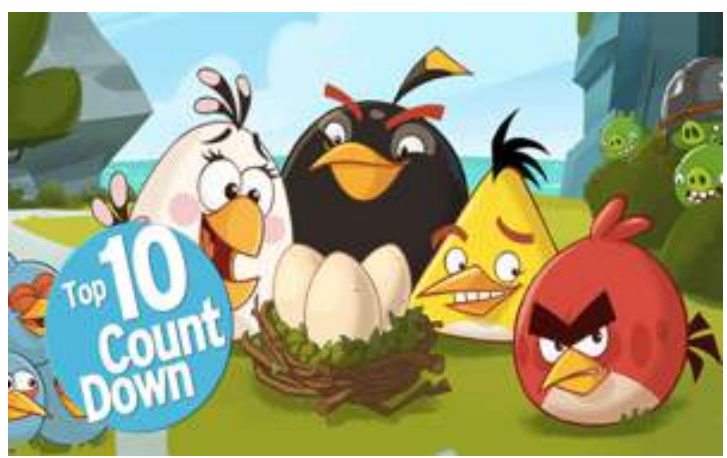

Figure 10 Casual Games (https://www.angrybirds.com/)

A god game is an artificial life game that casts the player in the position of controlling the game on a large scale, as an entity with divine and supernatural powers, as a great leader, or with no specified character (as in Spore), and places them
Vol. 01, No. 02, 2016

in charge of a game setting containing autonomous characters to guard.

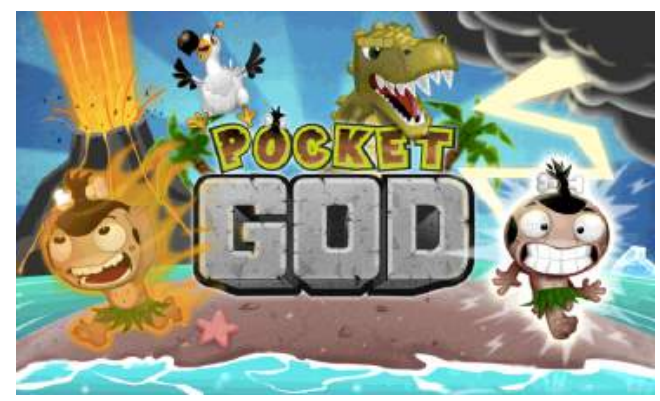

Figure 11 Pocket God (http://www.boltcreative.com/games/pocket-god/)

Educational games are games that are designed to help people to learn about certain subjects, expand concepts, reinforce development, understand an historical event or culture, or assist them in learning a skill as they play.

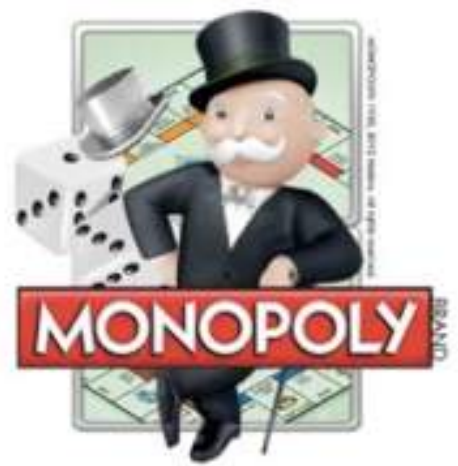

Figure 12 Monopoly (http://www.hasbro.com/enus/brands/monopoly)

Puzzle video games are a genre of video games that emphasize puzzle solving. The types of puzzles can test many problem-solving skills, including logic, pattern recognition, sequence solving, and word completion.

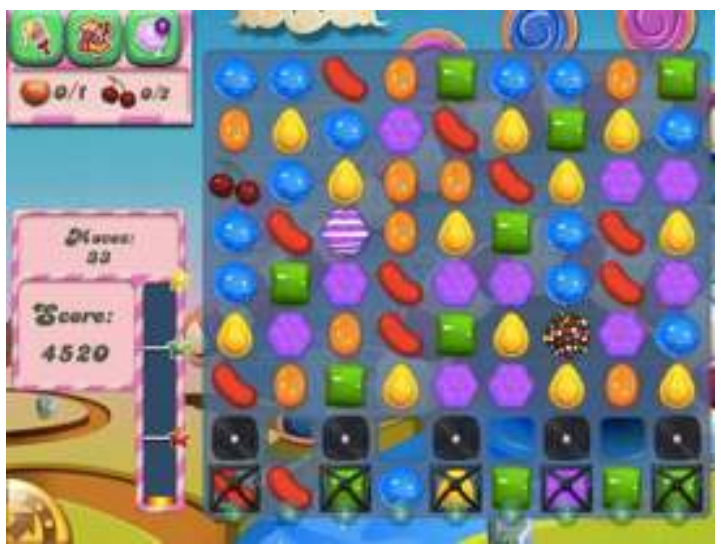

Figure 13 Candy Crush (https://king.com/game/candycrush)

An online game is a video game that is either partially or primarily played through the Internet or another computer network. 


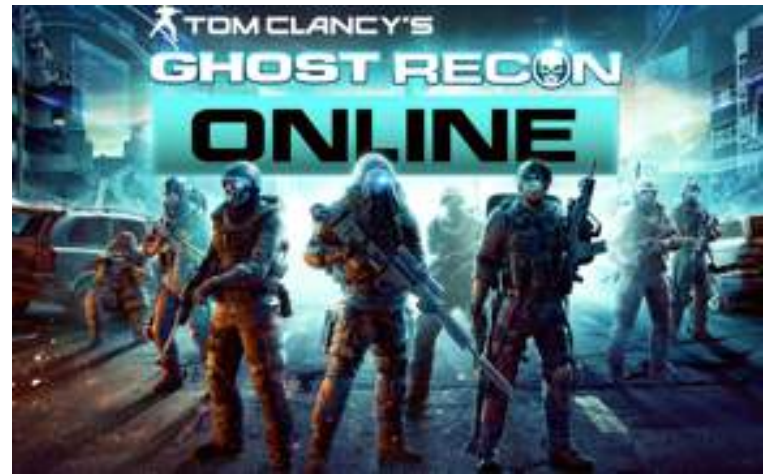

Figure 13 Tom Clancy's Ghost Recon Online (https://www.ubisoft.com/enGB/game/ghost-recon-phantoms/)

Through the concept development, the need to know what is client wants. If the client is looking for a blockbuster that will dominate the category, we must have to design a game that excels in all areas (requiring a larger budget and a long schedule). If the client wants a sequel to a franchise title in time for special day like Christmas or Independent Day perhaps you should concentrate on one or two new features and leave other portions unchanged because you know the timetable is inflexible. Sometimes we will be working on adaptation a port of an existing game to different hardware platform. This is usually a low-cost effort in which the client is trying to controller the investment from the initial game across as many platforms as possible. The key elements are to manipulate on this situation are:

- Good gameplay is the cheapest weapon in our collection, and we can wield it effectively on any project, no matter the schedule or budget.

- Scope, gameplay does not determine how expensive a project is or how long it takes.

Scope does: How big is the game? How many levels? How many creatures? How many weapons? How many tracks and cars do you put in a racing game? How many moves do you put in a fighting game?

- Technical risk is the success of your game will depend on the development of new technology, it will be more expensive, take longer to produce, and have a harder schedule to predict than a game that is built using existing technology.

Games are created opportunities for various organizational needs in industry, such as: accelerating learning [1], driving workforce productivity [2], communicating with customers $[3,4]$ and collaborating with business partners [5]. These game scan accelerate learning by creating 'flow' conditions [6], and thus increasing the engagement and the immersion of the participant $[7,8]$. The key elements of such games involve rules of motivation, known as Self-Determination Theory (SDT). SDT focuses on three interrelated categories: mastery, relatedness and autonomy, which address the need to allow innate growth and wellbeing tendencies to flourish [3, 9]. Mastery concept in business games refers to the degree of actual interaction between the player and in the game [8]. The mastery of control over tasks allows optimal performance, where people are gravitating to tasks, time often passes quickly and self-consciousness dissolves [10]. People can develop skills through performing optimally challenging tasks [7].

Similarity concept in games refers to social connection and desire to interact with other people. It can also manifest itself as a desire for a higher purpose [3]. Business can create cohesion and mutual respect through sparkling meaningful social collaboration between members in the context of the competition. Relatedness creates opportunities for the company to remove negative elements such as anxiety and fatigue from traditional forms of work. Instead, it creates a fun, engaging and interactive experiences that allow people to focus more on internalizing knowledge and work productively [3].

Autonomy concept in games refers to the innate need to feel in command of one's life and to be doing that which is meaningful [3]. Freedom to select what to do and how to perform aligns engagement with personal goals and offers an opportunity for interesting and stimulating work [7]. Games create autonomy by design tasks that are optimally challenging and rewarding. Games bridge simulation with entertainment, and can be personalized in for different learning styles. Managers can take pieces of the game elements and embed them in their progressions to suit their goals. Werbach and Hunter [3] suggest managers can and should tweak game elements to align their experiences with the firm's objectives.

On the flip side, games developers face many challenges. Managers can be distracted by glossy promises of games and miss deep challenges in game designs that can create value to the organization [11]. Promoting games needs support from top executives and effective top-down communication process [2]. The name of the word 'game' may be subjective in the corporate world. Managers may perceive games as playful activities [3]. Designing games that are both fun and educational can be difficult. People are attracted to games for entertainment purposes, which require games to be both educational and fun. Design of games requires a combination of experience, the artist's touch, and the time and financial support to make progress [12].

This article reviews the evidence base regarding the benefits of serious games when applied in business backgrounds. Categorizing the results to clearly define the concept of a serious game in a business context, reflects on evidence regarding their benefits and drawbacks and puts forward methods for their design and evaluation. In accomplishing, the overall findings of the review are considered with respect to their implications for businesses seeking to take advantage of serious games and gamification principles.

\section{PROBLEM ANALYSIS}

The approach taken to consolidate the available literature on the use of games in business contexts. This is a broad area, and consequently refines the scope of the review, presenting the key research questions used to highlight the review. The search strategy detailed in was then applied to identify papers relevant to these key research questions. The target of the 
research presented in this paper has been to identify, interpret and summaries the literature currently available on serious games, relating it to the needs of decision-makers and recognizing how best to support them in evaluating whether a game or gamification approach is relevant to a specific challenge. In scoping this study, the focus has been on articles that are central and relevant to serious games within manufacturing and business contexts. In terms of research questions, we approached this study by posing the following questions:

- What are the leading examples of serious games for business context?

- What are the benefits, limitation, inhibitors and enablers of serious games?

- What design methodologies can be applied to develop a successful serious game?

The purpose of these questions was to guide the search, with the authors being mindful that existing literature may be insufficient to allow these to lead to conclusive findings; however, identifying the shortfalls in the current evidence base allows for some important areas for future work to be identified, addressing question.

\section{SEARCH STRATEGY}

The strategy was developed by first identifying the relevant data sources, time frame and key words. Initially a very broad selection of databases was identified, to cover a diverse range of publication formats including journal articles, conference proceedings, theses, books, and articles from trade journals. These databases included IEEE Explore, ACM, and Inspect, Science Direct database along with the more traditional library cataloguing systems providing access to a variety of journals/conferences.

The search strategy initially identified key words that could be associated with Gamification. Examples of these include: serious games, gamification, business games, and game based learning. Initially this study focused on literature published between 2001 and 2013, with their citations being cross-checked to ensure any earlier publications were also captured. The principal research databases were then searched using a range of combinations of these key words. The lists of hits for each search string were firstly edited to remove any duplicate records that appeared, the titles were checked to ensure relevance to the review, and then the abstracts of all other articles and papers were reviewed before selecting publications for a full review. For completeness, an Internet search was also conducted using a similar process to that used with the library databases.

\section{RESULTS AND ANALYSIS}

Initially the search terms identified some 100 articles, reports and theses. These were then carefully filtered to establish 30 documents that were directly relevant to our research enquiry. The analysis itself was aided by applying mind-mapping techniques to capture and cluster the main themes and contributions.

\section{APPLICATION AND PENETRATION OF SERIOUS GAMES TO BUSINESS DECISIONS}

Before considering how games are perceived, it is essential to clarify their explanation in the context of this article. "Serious Games" represent a dramatic convergence of games and e-learning technologies to provide some rich, immersive virtual learning environments. By combining sophisticated theories of education with cutting-edge technology, serious games have tackled a wide range of challenges ranging from corporate training and education through to emergency medical response. The broadest definition of a serious game, therefore, is perhaps best defined as a game played for a purpose other than entertainment. Zyda [13] provides a broad-stroke definition of a serious game as "a mental contest, played with a computer in accordance with specific rules that uses entertainment to further government or corporate training, education, health, public policy, and strategic communication objectives'. Serious Games are games designed with the purpose not just to entertain, but to also solve a problem.

Bogost [14] in his book "Serious Games" defines them as games that "have an explicit and carefully through-out educational purpose and are not interned to be played primarily for amusement". In comparison to Gamification, that uses some design elements from games, Serious Games involve any application of the wider ecology of games [15]. For purposes of simplicity in this article, the term Gamification will be used to describe both Gamification and Serious Games. Today the use of game elements, design and mechanics is incorporated in many aspects of our lives such as education, work, entertainment, communication and exercise. Many researchers have studied the benefits of participating in games in peoples' lives. For example, Jane McGonical [16] mentions numerous aspects that can be promoted through games. Some examples are: motivation, competitiveness, collaboration, creativity, enjoyment, engagement, satisfaction and innovation. Many of researchers (i.e. [17-20]) support her claims and provide evidence that games have the capability of satisfying a range of needs found in [21] hierarchy of needs (e.g., creativity, problem solving, morality, spontaneity, self-esteem, confidence, achievement, respect of others, respect by others, friendship, family).

This can vary substantially between individuals and organizations: whilst evidence detailed in this article shows games can be a useful productivity tool, it is equally true that some employers have concerns regarding the intrusion of entertainment games into the workplace through both desktop PCs and mobile devices, distracting employees and reducing productivity. The potential to satisfy both stakeholders: the employer sees productivity gains through employees who are highly engaged with interactive and entertaining tools or training media, and the employees themselves gain both the educational benefits of more interactive training, and the motivational benefits of likeable gameplay - making work "fun" need not mean sacrificing productivity; in fact, it can enhance it. Reeves and Read [22] claim that games can 
enhance the overall productivity of employees by boosting collaboration, engagement, creativity, analytical thinking, quick decision making and many other success factors. Researchers [19, 23] have also provided guidelines and developed frameworks for successful and meaningful gamification of real life activities. Numerous businesses have employed gamification to achieve their goals, whether these are enhancing the user experience [24], boosting motivation [25] or promoting engagement [26]

As with any emerging medium, serious games initially represented a high-cost solution, requiring both investments in high-quality artwork, as well significant low-level programming expertise to translate a game design to a finished product. However, as a recent review of game engines for serious purposes demonstrated [27], the creation of tools and environments to support game development have increasingly allowed these costs to be reduced, as has an expanding market for pre-developed game content such as 3D objects, images, and functions. The technological advancements of the last two decades have not only allowed for the creation of sophisticated virtual worlds, but also substantially reduced the costs incurred by their development and deployment. As Internet access became prevalent amongst households in the developed world, it granted access to these virtual worlds to more than two billion visitors. Ryan, Rugby and Przybylski [28] characterize these virtual worlds as immersive, engaging and with increased complexity, and believe that they can be the enablers of numerous social behaviors, activities and goals. Bogost [29] believes that the interactive video games can set the stage for meaningful expression and persuasion, and are often considered an engaging and immersive solution.

The effectiveness studies which focus on measuring the impact of serious games on metrics such as engagement, motivation, and reflection - rather than comparing them to existing teaching methods - are particularly relevant, such as the evaluation of Triage Trainer conducted by de Freitas and Jarvis [30]. This evaluation presented an increasing in learning transfer when comparing serious games with a tabletop game as part of learning. Studies such of that of Mansoor and ElSaid [31] have shown that serious games can offer a level of social interaction like face-to-face contact, but without the physical restrictions and costs normally imposed by realworld reconstructions of training scenarios.

Rather than promoting serious games as a replacement or alternative to traditional learning methods, it is far more accurate and beneficial to stress their potential as a powerful complement to existing learning approaches. This is evident as the principal approach in references throughout this section: seldom is a game intended to fully replace an existing training programmer. That the use of a serious game can replace elements of existing courses effectively, being blended into other materials. Hence, whilst return on investment (ROI) might be seen from the greater impact of a training intervention with game-based components, rather than its reduced costs, a developed game can offer cost-reduction benefits due to the ease with which it can be distributed, and its capacity for replacing costly simulative or trainer- led components of a course. Serious games allow learning practitioners to offer previously difficult to deliver levels of interactivity, dynamism, and feedback to large groups of learners. By successfully addressing the challenge of creating games that are simultaneously compelling and educational, serious games developers are capable of motivating and challenging learners as they explore situations that are impractical or impossible to replicate using existing teaching methods.

As with any innovative solution, demonstration of value and appraisal of success hinges on the ability to prove ROI, which in turn incurs a need for research or clear metrics for impact, which may be difficult to ascertain when looking at long-term impact on behavior. Because of their ability to motivate, engage and influence behaviors, serious games are being used in the corporate sector for training, recruitment and marketing and sales, via targeting planning, problem solving and hypothesis verification. However, to improve the uptake and the evaluation of serious game, it is necessary for the designers to support higher order thinking (i.e strategic thinking, analysis and interpretation of events, preparation of research questions) and creativity simulation. This can be achieved through the advances in Artificial Intelligence (AI), concerning the simulation of (single) human behavior are needed, to allow creation of living worlds, populated with realistic or at least credible non-player characters (NPCs) (these NPCs are especially necessary for complex environments, in particular related to human sciences and the impact of technologies- refer to section 3.4) [78].

In the next paragraphs the authors are going to present games that were funded by large commercial organizations for branding and educate customer. Organizations are finding that the application of a game-based learning approach to corporate training is helping them increase employee engagement and drive performance over and above that previously delivered by traditional training approaches. Serious games provide employees with a compelling contextrelevant storyline, achievable goals, constant feedback on their progress and rewards such as achievement badges and public recognition. They also provide customer with opportunities to fail learn from their mistakes and try again in safe environments. Typical examples games used for are:
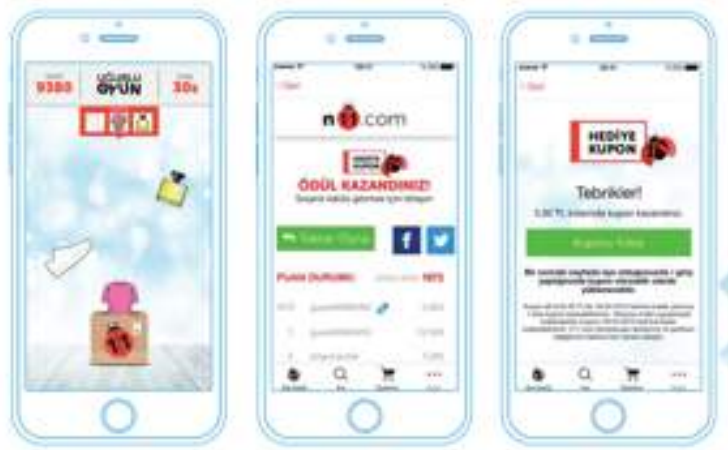

Figure 14 N11.com increased sales

(http://digitalmarketingmagazine.co.uk/digital-marketing-content/4businesses-that-are-using-digital-games-in-their-content-marketing-andwhat-happened/2682) 
N11.com is a popular online shopping portal. They used game content to engage and entertain users on their app. N11 also used the game as part of their customer loyalty program and to promote the latest products. The game got players to catch promotional products in their shopping bag to receive points and a discount coupon. $74 \%$ of unique users downloaded the discount coupon after engaging with the mini game. Takeaway: games can be used to add fun to delivering a coupon and build loyalty.

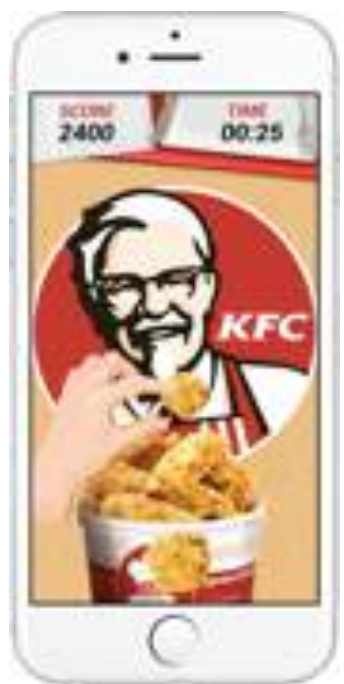

Figure 15 Kentucky Fried Chicken converted online fans into store visits (http://digitalmarketingmagazine.co.uk/digital-marketing-content/4businesses-that-are-using-digital-games-in-their-content-marketing-andwhat-happened/2682)

KFC Indonesia held an event in stores - the 'Hot Bucket Challenge'. KFC wanted a mobile game to generate buzz and awareness of the actual event happening in-store. The game involved eating chicken and got customers to visit the store and take part. In addition, $36 \%$ of players provided their contact details to KFC after playing the game. Takeaway: games can drive awareness of a promotion and drive players into stores. You don't need to be big, or have lots of money to use games as content and adverts. Lots of businesses are now taking advantage of games to boost engagement and conversions in their marketing.

Kentucky Fried Chicken and N11.com, a serious game, as an online marketing tool to showcase its products and services. It also uses the game as educated customer how to order and buy. This games gives players the opportunity and challenge of running a virtual shop or restaurant, complete with evaluation of product, quantity, price and the ability to improve process efficiency with the purchase and delivered to their home or office.

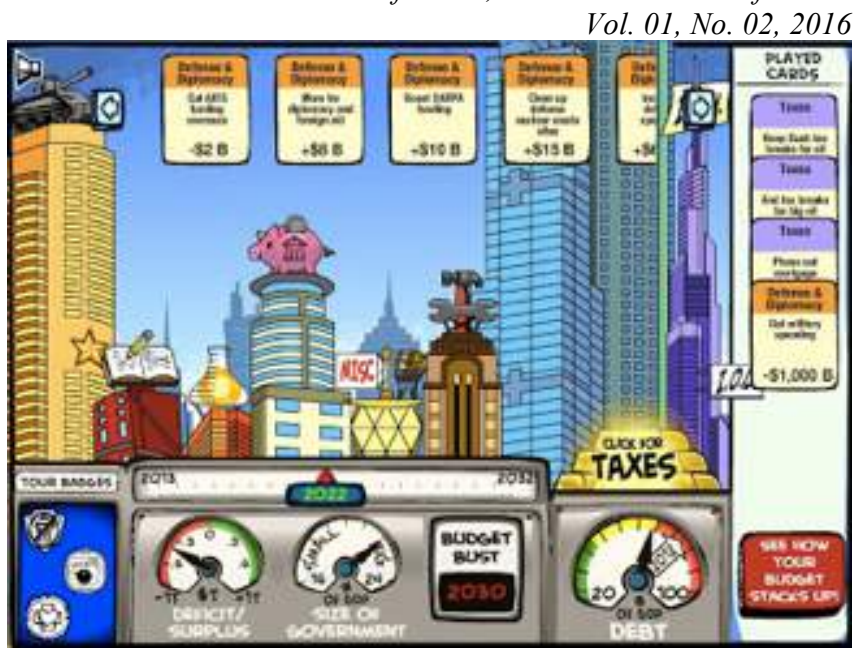

Figure 16 Budget Hero

(http://seriousgamesmarket.blogspot.co.id/2013/08/serious-games-forjournalism-playing.html)

Budget Hero by American Public Media In 2008, American Public Media published this simulation, which asks players to build a federal budget that can stay balanced over the next thirty years.

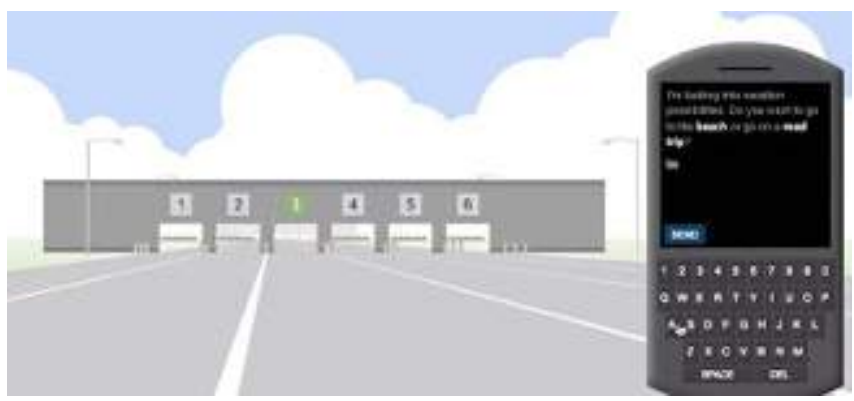

(http://seriousgamesmarket.blogspot.co.id/2013/08/serious-games-forjournalism-playing.html)

Gauging Your Distraction, by the New York Times. The game asks players to drive and text simultaneously, and requires you to do both well. Throughout the game, players must maneuver between six lanes as directed, all while texting correctly spelled messages back to a friend. 


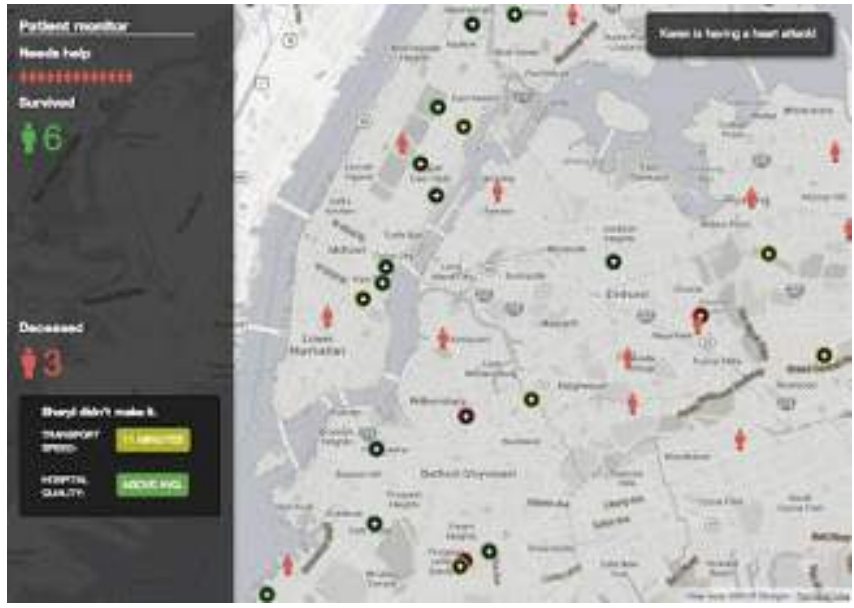

Figure 16 Gauging your Distraction

(http://seriousgamesmarket.blogspot.co.id/2013/08/serious-games-forjournalism-playing.html)

Newsgame HeartSaver by Sisi Wei. Players are shown a map of New York City, and are tasked with getting as many heart attack patients to emergency care facilities as possible. The game used the best data available, including querying the Google Transportation API for real transportation times between two locations, and data from the Emergency Medicine Journal on survival rates for heart attack patients. Finding 1: Serious games are being used for training, and educated in many areas such as healthcare, manufacturing and the public sector. It is allowing the employees and customer of the company to be rewarded and challenged.

\section{ENABLERS AND INHIBITORS FOR SERIOUS GAME}

In the previous section clearly demonstrates the benefits of effective serious games, several keys enabling and inhibiting factors have also been shown to affect their uptake. This in turn can make it challenge to construct a clear business case for a serious game, when compared to a more formal training approach which can build upon a larger corpus of research. However, as a recent business training review suggested, when seeking to address such goals as behavioral or cultural change within an organization, distributing resources across multiple interventions is likely to yield more success than a single intervention approach [34]. In such a context, a gamebased learning approach holds clear potential as an innovative approach to tackling a problem, able to communicate concepts in novel ways whilst holding a unique form of appeal for target audiences.

As games, have frequently been show to work effectively as part of blended learning approaches [35], their potential for integration alongside other forms of learning material has enabled their use as a supplement and enhancement [36], rather than replacement, for existing training programmers. The ability to support and scaffold game-based learning with external materials also enables more flexible game designs, which can focus on delivery of the content and concepts best suited to game-based learning, rather than attempting to convert an entire training programmer to a game-based form. This allows games to focus on higher levels of Bloom's taxonomy [37], allowing players to apply knowledge and experientially learn, rather than attempt to communicate information in isolation.

Emergent game- development environments are increasingly challenging this presumption, allowing game developers to rapidly and efficiently build serious games [27]. Hence whilst cost could be an inhibitor, the emergence of technologies to streamline the development of games and rapidly create structured, immersive game based content has proven a strong enabler. The existing cultures and perspectives on gameplay are a final consideration as both enabling and inhibiting factors. Whilst a recent ISFE report showed over half of European respondents to consider themselves "gamers", the stereotype of the gamer as a young male persists in some sectors [38]. Whilst a wide range of both serious and entertainment games successfully targeting other groups have challenged this assumption, the acceptance of individuals at all levels of an organization's hierarchy of the benefits and potential of game based learning is a key enabling factor.

A study of the serious game "Ward off Infection", for example, showed that for hospital wards where senior management struggled to perceive the benefit of the game, this was transferred to front-line staff who subsequently failed to engage [39]. Buy-in at all levels of an organization can be difficult to foster, particularly if senior management are not well-represented in stakeholder groups during the design phase of a game. However, a positive attitude at all levels of an organization has strong benefits in supporting the uptake of any new technology through the generation of perceived usefulness [40], including serious games.

Finding 2: Serious games benefit business decision making by engaging and motivating their workforce, improving guidance outcomes and influence the behavior of their existing and new potential customers, however the effectiveness of the serious games could be influenced by amount risk factors such as the rate of change of ICT Technologies and the ongoing efforts to support the infrastructure, losing the balance between pedagogy and gaming, the change in nature of gamers.

Finding 3: The growth in serious games is being enabled by the is a lack of empirical research relatable to specific business challenges, the growth of the gaming industry, the new generation of games, and by contrast is being inhibited by existing cultures and perspectives on gameplay, unwillingness by the senior manager to adopt the gaming culture.

\section{DESIGN PROCESSES SUPPORTING THE DEVELOPMENT OF SERIOUS}

This section, we review frameworks and methodologies for the development of serious games and consider how this relates to a business environment. On a technical level, a digital game is not dissimilar to any other large software development project, and therefore recognized models such as Boehm's spiral [41] are readily applicable. One perspective describes a serious game as an iterative, user-centric agile development project [42]; iteration is expressed as central in a range of methodologies for serious game development [43, 44]. However, in its loosest form, iteration can be suggested 
as a solution to a wide range of issues; the problem is translating the iterative cycle into one sufficiently pragmatic for game development within resource constraints. In doing so a range of unanswered questions emerge: if investing resources into multiple iterations results in a lower fidelity game, does it remain the optimum route in the face of research suggesting such fidelity is so valuable [27]? If we do iterate, how do we ensure each prototype is sufficiently well researched to ensure valuable feedback into the next cycle? Here games again present some unique considerations in terms of both the challenges and potential they afford when used as research instruments [45]. Also, noted in literature is the need for development effort to be genuinely collaborative in nature [46, 47], a consequence of the need to balance carefully the needs of engagement with the needs of instructional design [48].

The various stakeholders in a serious game development project are seldom co-located, a major factor in effective collaborative design [47]. Furthermore, the various perspectives of these stakeholders must be considered through objective research rather than subjective input, else a game can risk duplicating existing problems [49]. Similarly, a risk may exist of games being designed to meet stakeholder expectations, taking a simulative route due to the ease in aligning the look-and-feel of the game with that of more conventional educational material. Simulation is partly paradigmatically opposed to gaming: simulations strive for reality, whereas games will readily sacrifice it if it becomes a barrier to user experience. Evidence comparing high- fidelity simulations to lower-fidelity games has demonstrated results in favor of the more engaging experience. Social games present a challenge from this perspective, as the game may function more as a tool for populating and sustaining a social network, rather than an instructional medium. As such, an entertainment game could serve as an effective "serious" tool, with its key defining characteristic being its owner, rather than its content. Reports from pragmatic development contexts reinforce these concerns [50].

In addition to over- prescription of iteration and reluctance to embrace fully a game-based medium, Werneck and Cheng report other issues to include negotiation within the project, level of scrutiny imposed to more novel approaches, previsioning and postponement, and misinformation on resources. These alone each represent significant barrier in attempting to enact a collaborative development project, even more so when cast in the light of negative perceptions of gaming still noted in some sections of the organizational hierarchy by this study. It is important to consider this study in terms of the single case it reports on, but other studies have similarly reported difficulties in serious game development to arise from the complex multi-organizational structure at the core of many projects [51], as well as the constraints of technology, domain knowledge, user research, and game design. This is reiterated from an alternative perspective in the four-dimensional framework [52], which posits learners, their context, the representational medium, and pedagogic method to be key, though offers little guidance beyond highlighting these initial considerations due to the lack of an evidence base on which to construct such guidelines.
Pervasive and mobile computing offers some potential to move beyond these confines and create new models and mediums for learning transfer. Physical activity is an obvious area for this application which has been explored through several systems with positive outcomes [53]. Sensor networks and virtual worlds have also been explored towards more general learning objectives with promising early findings [54]. Frameworks in support of the development and deployment of games in pervasive and mobile contexts are emerging, and though again lacking in conclusive demonstrations of efficacy, provide some relevant considerations. To prescribe a framework for persuasive gaming, Oja and Riekki focus primarily on the case of ubiquitous games, noting the importance of access to data and considering both bespoke games and gamification [55]. Omitted, however, are the underlying ethical questions raised when seeking to adjust behavior, and particularly how this access to data can be achieved consensually without compromising the efficacy of the intervention: if we inform users of the purpose of the activity to inform consent, we might similarly compromise its efficacy as a means of "stealthy" learning transfer [56].

Finding 4: Effective serious game development requires involvement from stakeholders throughout the development process.

\section{TECHNOLOGIES SUPPORTING THE DEVELOPMENT OF SERIOUS GAMES}

The technical state-of-the-art in serious games mirrors that of leisure games [57], however the technical requirements of serious games are frequently more diverse and wide ranging than their entertainment counterparts. Serious game developers frequently resort to bespoke and proprietary development due to their unique requirements, such as [58] and [59], and difficulties exist for game engine developers in accurately understanding and supporting the needs of instructional design. The popularity of video games, especially among younger demographics, results in them frequently being perceived as an ideal medium for instructional programmers aimed at hard-to-reach audiences [37]. However, preliminary studies have also shown this demographic responds poorly to low-fidelity games [60], and as a result there has been a trend towards the development of more complex serious games that are informed by both pedagogic and game-play elements.

Although many serious games have limited visual interactivity, immersion and fidelity, there is an increasing motivation to create serious games that intend to support situated (social and peer-driven) and experiential pedagogies; partially because behaviorist approaches have been shown to be limited (e.g. people learn to play the game, not address learning requirements), whilst cognitive approaches struggle to impart deeper learning in the areas of affect and motivation [61]. Furthermore, recent work by Mautone [62] demonstrated enhanced learning when introducing game elements to a standard flight simulator. Consequently, re-evaluation of simulator approaches to incorporate game and game-like elements places an increasing demand for serious game developers to deliver high- fidelity solutions. Given this motivation to create immersive, high fidelity serious games, 
an obvious development choice is to utilize game engines, which provide 'out of the box' support for state-of- the-art desktop GPU rendering and physics.

One of the most important elements of the creation of serious games is the visual representation of these environments [63]. Although serious games have design goals that are different from those of pure entertainment video games, they can still make use of the wide variety of graphical features and effects that have been developed in recent years [64].

The creation of a serious game is a complex engineering project that requires skill and dedication. The development of a serious game engine is a complicated process that requires time, resources and teamwork. As serious games become more complex, so do the engineering challenges that arise during development of the game. Hence the selection of an ideal engine for this development is crucial. To simplify the game engine selection Petridis et.al have designed a framework which allows the developers of the serious game to select the most appropriate engine, based on the technical requirements and the instructional design of the serious games. Based on the framework the game engines are categorized according to their visual/audio fidelity, functionality, networking capabilities, composability and accessibility. Examples of such game engines that are currently used in $r$ corporate training are Unity and Sealund's Just PlayIt.

Tabel 1: Framework for comparing engines in SG

\begin{tabular}{|c|c|}
\hline \multirow{3}{*}{ Audiovisual Fidelity } & 'Rendering \\
\hline & "Animation \\
\hline & Sound \\
\hline \multirow{3}{*}{ Functional Fidelity } & Scripting \\
\hline & Supported AI Techniques \\
\hline & Physics \\
\hline \multirow[t]{2}{*}{ Composability } & Import/ Export Content \\
\hline & Developer Toolkits \\
\hline \multirow[t]{2}{*}{ Accessibility } & Learning Curve \\
\hline & Documentation and \\
\hline
\end{tabular}

\begin{tabular}{|l|l|}
\hline \multirow{2}{*}{} & \multicolumn{1}{|c|}{ Sol. 01, No. 02, 2016 } \\
\cline { 2 - 2 } & Licensing \\
\cline { 2 - 2 } & Cost \\
\hline Networking & $\begin{array}{l}\text { Client Server/ Peer-to- } \\
\text { peer }\end{array}$ \\
\hline Heterogeneity & Multiplatform Support \\
\hline
\end{tabular}

Finding 5: The technologies supporting the development of serious games has been advanced by the rapid evolution of technology in gaming and ICT industry in the areas of visual fidelity, artificial intelligent, haptic devices and sensors, networking, CPU advancements, etc, though future work should address the many issues surrounding the equation of the learning requirements to the technical features.

\section{EVALUATION METHODOLOGIES OF SERIOUS GAMES}

There are a few established generic evaluation approaches which can be used to evaluate serious games, however there are dependent between the sector for which the game is designed and the nature of the evaluation design. This presents something of a dilemma when attempting to provide a comprehensive review of evaluation methods: approaches such as randomized control trials [65, 66], focus groups [67], interviews, narrative inquiries, and quantitative analyses of game engine data [68] have all been conducted. As an emerging form of training medium, a strong argument exists that serious games should be evaluated exactly as any other educational medium, and affording them specific consideration with respect to their evaluation detracts from the comparability of any results. Therefore, the methodological toolkit of a serious game evaluator needs to be a broad one: often the sector dictates the most appropriate methodology, rather than the use of a serious game itself. Serious games present an advantage in that the game engine, could be analyzed to understand the player behavior and through relating this to real-world behavior [68]. Due to the difficulty in assessing factors such as motivation and behavior directly, emphasis has frequently been given to establishing proxy measures of efficacy, such as how realistic a simulation is through analysis of technological aspects of the humancomputer interaction [69]. However, Norling notes that believability is often not a paramount concern, and that excessive focus on this criterion can be to the detriment of games' ultimate goals [70]. Thus, transposing evaluation simulation methods to serious games is inadequate because the focus of the evaluation should be more focused on the educational content.

It has been shown that serious games must be able to exhibit effective learning transfer, whilst also engaging the user [71, 72]. Several studies have focused on the assessing the gameplay experience in isolation [73]. If a game cannot 
engage learners, then sourcing an adequate sample of experienced players with whom to assess learning outcomes becomes an impossible task [74]. We therefore go on to describe evaluation techniques with respect to these two key areas of engagement and learning transfer. Engagement has been measured in the medical area for applications such as stroke rehabilitation [24]. Burke et al. [24] identified game design principles for upper limb stroke rehabilitation and present several developed games using video-capture technology. In this case, the evaluation approach adopted a randomized control trial, which monitored usage between healthy subjects and stroke victims, showing positive early results. Heuristic approaches to evaluation also offer some potential [75]. Pinelle et al. by analyzing reviews of 108 games identified 12 common classes of usability problems, which lead them to the development of a set of ten usability heuristic based on the problem categories [76].

Several frameworks have been developed such as TILT and Flashlight to evaluate the integration of technology in teaching. However, these frameworks evaluate the integration of the technology in teaching rather than learning.

Such a perspective can be difficult to apply for serious games, particularly those distributed online or in an e-learning context where the presence of the tutor cannot be relied upon. Few frameworks specifically delineate methods for gamebased learning, understandable, since any such evaluation benefits from its ability to be compared methodologically and in terms of results to other learning solutions. Qualitative work has been used extensively to assess serious games, though it is easy to argue its selection is often grounded more in pragmatism than suitability. Certainly, qualitative work can be essential in providing insight into learner response and understanding, and when conducted rigorously can form a core basis on which to build structural models for quantitative assessment. However, qualitative findings alone, particularly with a limited sample size, are often one of the central criticisms of inadequate serious game evaluations.

Finding 6: Evaluation methods need to be broadened to elicit deep understandings of game efficiency and their impact on learning. Although until now it is evidence that quantitative / statistical methods are often being used in evaluating a serious game, we argue that more qualitative evaluations would be necessary to discern qualitative differences in conceiving and approaching a serious game.

Finding 7: We perceive that the consistent use of such methodologies will help game and learning designers as well as game researchers to understand better what players do in games and thereby becoming better at designing adaptive games and effective game frameworks that transform learning experiences.

\section{Discussion}

Learning through play is not a new concept. Educational computer games (i.e serious games), underpinned by pedagogical goals and the appropriate use of game mechanics have potential for learning through the intrinsic ability to engage, motivate and could influence the behaviors of the users. Games through storytelling, quests, rewards and competitions can create an environment in which learning could take place. Large organization such as IBM, Microsoft, Dell, Cisco, etc are using serious games to train their workforce from compliance training to leadership training. Well-designed serious games can make learning fun, challenging and rewarding. Serious games designers are faced with the challenge of designing a game which is fun, tided up with pedagogical elements [13].

The methodology, then, must safeguard against both these failure conditions: on the one hand, it must ensure the game retains the engaging characteristics that make game-based learning an optimal selection for the learning context; on the other, it must ensure that effective pedagogy is implemented in a synergistic fashion with gameplay elements. Thus, the importance for more formal design methodologies for serious games is well documented [77].

A central challenge in creating a prescriptive approach is being able to sufficiently evidence context-independency of development models, since a proven approach for one serious game may not be applicable to another, given the broad range of topic areas and learner demographics games. Existing elearning development methodologies have met limited success when transposed to serious gaming, as they emphasize instructional content with little affordance for the unique way in which games attract and retain learners.

To create a successful serious game, we need to ensure involvement from stakeholders throughout the development process. Therefore, care should be taken to support stakeholder involvement regardless of development context, supporting where possible co-location and open channels of communication between all parties.

Ultimately, the design and implementation of effective serious games must be grounded in pedagogy, as well as technology, and therefore future work should address the many issues surrounding the equation of learning requirements to these identified technical features. Towards this end, future studies will focus upon the analysis of the impact of the various engines and their functionalities on targeted learner groups.

Evaluation methods need to be broadened to elicit deep understandings of game efficiency and their impact on learning. Although until now it is evidence that quantitative / statistical methods are often being used in evaluating a serious game, we argue that more qualitative evaluations would be necessary to discern qualitative differences in conceiving and approaching a serious game. Playing a serious game predominantly generated a subjective experience, which is based on individual beliefs, conceptions, and actions that are being evoked in a different manner encompassing novel game plays and game mechanics. There is a need to collect, capture and analyze these conceptual artifacts in relation to the affordances of games to better map the efficacy of game elements to individual experiences. This may be achieved through applying methodologies that have already been used and proved their rigout in educational research such as phenomenography, grounded theory and action research among others. We perceive that the consistent use of such 
methodologies will help game and learning designers as well as game researchers to understand better what players do in games and thereby becoming better at designing adaptive games and effective game frameworks that transform learning experiences.

In congruence with this, game frameworks that align game play/mechanics with teaching methods, learning styles, feedback and assessment processes would facilitate the process of developing and evaluating complex learning features in games that would literally add the proliferated educational value in a serious game.

\section{CONCLUSIONS}

This article has presented a structured review of the literature on the use of serious games in a business context. Whilst these benefits in turn have contributed to increased uptake of game-based approaches, serious game developers need to be aware of the need for solutions to provide demonstrable return on investment and solutions to business needs.

The increasing evidence base is already challenging perceptions that work cannot be "fun", and the use of games and gamification principles has demonstrable potential to improve the efficacy of training programmers, increase productivity, and even reach out to a global community of volunteers willing to contribute their time to gamified problem-solving.

The next step will include the implementation of a framework for the creation and evaluation of serious game for business context and especially in the sterilizations area.

\section{REFERENCES}

[1] Laine, T., Using a business game concept to enhance servitization: a longitudinal case study, Managing Service Quality, 2012, 22: p. 428-446. http://dx.doi.org/10.1108/09604521211281369

[2] Cook, W., Five Reasons You can't Ignore Gamification. Chief Learning Officer 2013. 12(5): p. 46-55.

[3] Werbach, K., and Hunter, D., For the Win: How Game Thinking Can Revolutionize Your Business. 2012: Wharton Digital Press.

[4] Zichermann, G.L., J., Game-Based Marketing: Inspire Customer Loyalty Through Rewards, Challenges, and Contests. 2010: Wiley.

[5] Hugos, M., Enterprise Games: Using Game Mechanics to Build a Better Business, . 2012,: O'Reilly.

[6] Csikszentmihalyi, M., Beyond Boredom and Anxiety: Enjoyment and Intrinsic Motivation. 1975, London (United Kingdom): Jossey-Bass Inc.

[7] Mainemelis, C., When the muse takes it all: A model for the experience of timelessness in organizations, Academy of Management Review, 2001, 26, p. 548-565. http://dx.doi.org/10.2307/3560241

[8] Bedwell,W.L.,Pavlas,D.,Heyne,K.,Lazzara,E.H.\&Salas,E., Toward a Taxonomy Linking Game Attributes to Learning: An Empirical Study, Simulation and Gaming, 2012, 43, p. 729- 760. http://dx.doi.org/10.1177/1046878112439444

[9] Deci, E.L.R., R. M., Handbook of Self-determination Research,. University of Rochester's Press., 2004.
[10] Pink, D.H., Drive: The surprising truth about what motivates us, Canongate, 2010,

[11] Shelton, T., Business Models for the Social Mobile Cloud: Transform Your Business Using Social Media, Mobile Internet, and Cloud Computing, 2013: John Wiley \& Sons. http://dx.doi.org/10.1002/9781118555910

[12] Edery, D.M., E., Changing the Game: How Video Games Are Transforming the Future of Business, 2008: FT Press.

[13] Zyda, M., From visual simulation to virtual reality to games. IEEE Computer, 2005.

[14] Bogost,I., Procedural Retorics' in Persuasive Games: The Expressive Power of Videogames., ed. Anon. 2007. 1-64.

[15] Deterding, S., Khaled, R., et al. , Gamification: Towards a Definition. CHI, 2011

[16] McGonigal, J., Reality is Broken: Why Games make Us Better and how they can Change the World. 2011, New York,: The Penguin Press.

[17] Gee, J.P., What video games have to teach us about learning and literacy. 2003: New York: Palgrave Macmillan.

[18] Zhang, P., Motivational Affordances: Reasons for ICT Design and use. Communications of the ACM, 2008. 51(11): p. 145147. http://dx.doi.org/10.1145/1400214.1400244

[19] von Ahn, L., Dabbish, L., Designing Games with a Purpose. Communications of the ACM, 2008. 51(8): p. 58-67. http://dx.doi.org/10.1145/1378704.1378719

[20] Sánchez, J., and Olivares, R.. Problem Solving and Collaboration using Mobile Serious Games'. Computers \& Education 2011. 57(3): p. 1943-1952. http://dx.doi.org/10.1016/j.compedu.2011.04.012

[21] Maslow, A.H., Motivation and Personality. 1954, New York, Harper and Row. 